\title{
Momentum dependent relaxation rate and pseudogap in doped magnetic insulators
}

\author{
M. M. Zemljič ${ }^{1}$ and P. Prelovšek ${ }^{1,2}$ \\ ${ }^{1}$ J. Stefan Institute, SI-1000 Ljubljana, Slovenia and \\ ${ }^{2}$ Faculty of Mathematics and Physics, University of Ljubljana, SI-1000 Ljubljana, Slovenia
}

(Dated: 16th June 2018)

\begin{abstract}
The spectral functions and corresponding self energies are calculated within the planar $t-t^{\prime}-J$ model as relevant to hole-doped cuprates using the exact diagonalization method at finite temperatures, combined with the averaging over twisted boundary conditions. Results show truncated Fermi surface at low doping and $t^{\prime}<0$ in the antinodal region while the self energy reveals weakly $\mathbf{k}$ - and doping dependent anomalous relaxation rate $\left|\Sigma^{\prime \prime}(\mathbf{k}, \omega)\right| \sim a+b|\omega|$ for $\omega<0$, consistent with recent ARPES results, and a pseudogap-generating component of Lorentzian form. The latter is well pronounced at low doping and strongly depends on $\mathbf{k}$ and $t^{\prime}$.
\end{abstract}

PACS numbers: 71.27.+a, 75.20.-g, 74.72.-h

It is well established that high- $T_{c}$ superconductivity in cuprates develops directly from a very anomalous normal state, which does not follow the usual Fermi-liquid (FL) scenario and thereby the concept of well defined quasiparticle $(\mathrm{QP})$ excitations. The nature of the latter is most directly accessed by angle resolved photoemission spectroscopy (ARPES), which probes the single-electron spectral function (SF) $A(\mathbf{k}, \omega)$ [1]. Concentrating here on the models for hole-doped cuprates, we furtheron discuss several ARPES normal-state results. a) Upon doping, the insulating antiferromagnet (AFM) develops into a metallic paramagnetic state where the Fermi surface (FS) appears as a large one, consistent approximately with the Luttinger FL volume in $\mathrm{La}_{2-x} \mathrm{Sr}_{x} \mathrm{CuO}_{4}$ (LSCO) [2] or evidently deviating from it in $\mathrm{Ca}_{2-x} \mathrm{Na}_{x} \mathrm{CuO}_{2} \mathrm{Cl}_{2}$ (Na-CCOC) [3]. b) The FS and corresponding QP are well pronounced only along the nodal direction at $\mathbf{k} \sim(\pi / 2, \pi / 2)$, while FS is truncated or poorly defined towards the antinodal points $\mathbf{k} \sim(\pi, 0)$, where the pronounced feature is the large pseudogap first observed in optimally-doped $\mathrm{Bi}_{2} \mathrm{Sr}_{2} \mathrm{CaCu}_{2} \mathrm{O}_{8+\delta}$ (Bi2212) [1, 4] and well developed in low-doped LSCO [5, 6] and Na-CCOC [3]. c) The QP relaxation rate, best identified along the nodal direction at optimally doped Bi2212 [1, 7], follows the non-FL behavior $\left|\Sigma^{\prime \prime}(\mathbf{k}, \omega)\right| \sim b(|\omega|+\zeta T)$, as summarized within the marginal FL concept (MFL) [8]. Recently, it has been analyzed all along the FS and represented by $\left|\Sigma^{\prime \prime}(\mathbf{k}, \omega)\right| \sim$ $\tilde{a}_{\mathbf{k}}+\tilde{b}_{\mathbf{k}}|\omega|$, where $\tilde{a}_{\mathbf{k}}$ is strongly momentum dependent and large in the antinodal part of the FS, while $\tilde{b}_{\mathbf{k}}$ is nearly a constant [9].

Above experimental facts still represent the major challenge in the theory of correlated electrons. There are by now several numerical results confirming that prototype models as the Hubbard model and the $t-J$ model on a square lattice can account for such phenomena. Pseudogap behavior within the Hubbard model has been observed in the quantum Monte Carlo (QMC) studies [10], and more recently using dynamical cluster approximation (DCA) [11, 12] and cellular dynamical mean-field theory (CDMFT) [13, 14] revealing its presence in both $A(\mathbf{k}, \omega)$ and density of states (DOS) $\mathcal{N}(\omega)$. Within the $t-J$ model as relevant to cuprates, the numerical study of SF using the finite temperature Lanczos method (FTLM) established the non-FL behavior of $\Sigma^{\prime \prime}(\mathbf{k}, \omega)$ as well as the pseudogap in DOS [15, 16]. More recent studies detected also the sensitivity of pseudogap features to the addition of next-nearest neighbor hopping $t^{\prime}$, both in the $t-J$ model [17] and the Hubbard model [11, 14]. In spite of the accumulated evidence, there is still no consensus on the origin of non-FL behavior and pseudogap. However, several numerical studies [10, 11, 13, 15, 16] and also analytical approaches [18] suggest the short-range AFM fluctuations as the generator.

In order to distinguish between different scenarios and make a comparison with ARPES experiments, more detailed knowledge about the momentum dependence of $\Sigma^{\prime \prime}(\mathbf{k}, \omega)$ is needed. In this Letter we present the results for the latter, as evaluated within the $t-t^{\prime}-J$ model, improving the FTLM with a continuous $\mathbf{k}$ variation on small lattices. We show that the self energy can be decomposed for $\omega \lesssim 0$ into an essentially k-independent MFL part $\left|\Sigma^{\prime \prime}(\mathbf{k}, \omega)\right| \sim a+b|\omega|$ and a pseudogap-generating contribution, which is strongly $\mathbf{k}$ dependent. We also find that the pseudogap part essentially depends on $t^{\prime}$.

Our study is devoted to the extended $t-J$ model

$$
H=-\sum_{i, j, s} t_{i j} \tilde{c}_{j s}^{\dagger} \tilde{c}_{i s}+J \sum_{\langle i j\rangle}\left(\mathbf{S}_{i} \cdot \mathbf{S}_{j}-\frac{1}{4} n_{i} n_{j}\right),
$$

where $\tilde{c}_{i s}^{\dagger}=\left(1-n_{i,-s}\right) c_{i s}^{\dagger}$ are projected fermionic operators not allowing the double occupancy of sites. We include on a square lattice besides the nearest- neighbor hopping $t_{i j}=t$ also the next-nearest-neighbor hopping $t_{i j}=t^{\prime}$. We consider here $t^{\prime}=-0.3 t$, as relevant for hole doped cuprates [17], as well as the reference $t^{\prime}=0$. In correspondence with experiments on cuprates we also fix $J=0.3 t$ and note that $t \sim 400 \mathrm{meV}$.

We calculate Green's function for projected fermionic operators,

$$
G(\mathbf{k}, \omega)=-i \int_{0}^{\infty} d t \mathrm{e}^{i(\omega+\mu) t}\left\langle\left\{\tilde{c}_{\mathbf{k} s}(t), \tilde{c}_{\mathbf{k} s}^{\dagger}\right\}_{+}\right\rangle,
$$

and the corresponding SF $A(\mathbf{k}, \omega)=-\operatorname{Im} G(\mathbf{k}, \omega) / \pi$, where $\mu$ is the chemical potential. In the following we present results obtained by using the exact diagonalization approach for 
small tilted (Pythagorean) square lattices with $N=n^{2}+m^{2}$ sites. Although we are primarily interested in the low $T \rightarrow 0$ regime, there are several advantages to perform the calculation at $T>0$, using the FTLM [15]. As discussed before [16], the spectra even on a small system become quite dense and macroscopic-like at $T>T_{f s}$, where finite-size temperature depends on the size $N$ as well as on the model used. Consequently, the finite-size effects are substantially reduced at $T>T_{f s}$. For our purpose it is important that in the latter regime we are able to extract a meaningful self-energy $\Sigma(\mathbf{k}, \omega)$ from the SF. In the following we present results for systems of $N=18,20$ sites ranging from low to intermediate doping, i.e. $N_{h}=1,2,3$ holes, where the corresponding $T_{f s}=0.1-0.15 t$. Within FTLM one evaluates separately the electron creation and annihilation SF $A_{+,-}^{N_{h}}(\mathbf{k}, \omega)$ representing the transitions $N_{h}^{\prime}=N_{h} \mp 1$, respectively. In fact, we calculate directly only $A_{-}^{N_{h}}(\mathbf{k}, \omega)$ and express $A_{+}^{N_{h}}(\mathbf{k}, \omega)=$ $\exp (\beta \omega) A_{-}^{N_{h}-1}(\mathbf{k}, \omega)$. Here we fix $\mu$ by requiring the total sum rule $(1 / N) \sum_{\mathbf{k}} \int d \omega A(\mathbf{k}, \omega)=\alpha=\left(1+c_{h}\right) / 2$.

On a system with periodic boundary condition (BC) one is able to consider SF only for a set of discrete $\mathbf{k}=\mathbf{k}_{\mathbf{l}}, l=1, N$. In order to scan the whole Brillouin zone, we employ twisted BC by introducing the uniform vector potential $\vec{\theta}$, which modifies the hopping elements $t_{i j} \rightarrow \tilde{t}_{i j}=t_{i j} \exp \left(i \vec{\theta} \cdot \vec{r}_{i j}\right)$ in the Hamiltonian, Eq. (1). It is well known that this procedure allows one to reach arbitrary momenta $\mathbf{k}=\mathbf{k}_{\mathbf{l}}+\vec{\theta}$. In the following we perform calculation for different phases $\vec{\theta}_{t}$, which are chosen equidistantly in the quarter of the first Brillouin zone (FBZ). In particular, our mesh contains $10 \times 10$ and $7 \times 7$ k-points for $N=18$ and $N=20$, respectively.

Using FTLM we calculate SF [15, 16] and extract corresponding self energies $\Sigma(\mathbf{k}, \omega)$. Due to non-trivial sum rule within the projected model, Eq. (1), the latter are defined via

$$
G(\mathbf{k}, \omega)=\frac{\alpha}{\omega-\zeta_{\mathbf{k}}-\Sigma(\mathbf{k}, \omega)} .
$$

Since we require that $|\Sigma(\mathbf{k}, \omega \rightarrow \pm \infty)| \propto 1 / \omega$, the 'free' term is uniquely determined as $\zeta_{\mathbf{k}}=\int d \omega \omega A(\mathbf{k}, \omega) / \alpha$. It should be noted that due to projection the $t-J$ model does not directly possess any 'free' band term therefore the dispersion $\zeta_{\mathbf{k}}$ is already nontrivial, dependent on doping $c_{h}$ and model parameters [18].

In order to reduce finite-size fluctuations of $\Sigma(\mathbf{k}, \omega)$ among different $\mathbf{k}$ we use additional averaging analogous to the treatment common in the cluster DMFT approaches. The underlying idea is that $\Sigma(\mathbf{k}, \omega)$, being quite local quantity in strongly correlated systems, varies with $\mathbf{k}$ more smoothly than corresponding $A(\mathbf{k}, \omega)$. Our results presented furtheron confirm this conjecture. Therefore, we perform the averaging of calculated $\Sigma(\mathbf{k}, \omega)$ (as well as $\zeta_{\mathbf{k}}$ ) using Gaussian weighting with radius $\delta k \sim 0.3$. Such averaged $\Sigma(\mathbf{k}, \omega)$ and $\zeta_{\mathbf{k}}$ are then inserted into Eq. (3) to evaluate SF.

First, we present in Fig. 1 results for the SF at the chemical potential, $A(\mathbf{k}, \omega=0)$, calculated with $t^{\prime} / t=-0.3$ at the lowest reachable $T=0.1 t \sim T_{f s}$, as a continuous scan
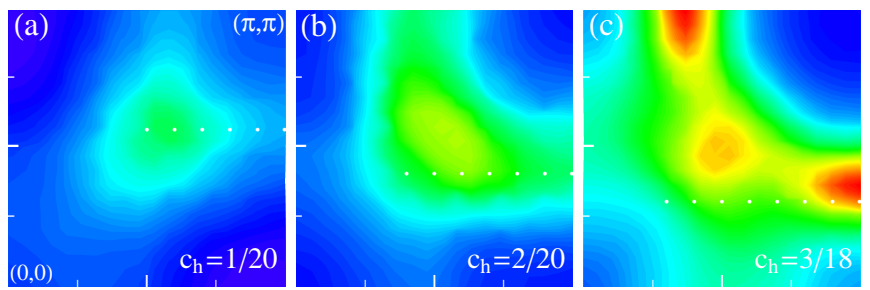

0

0.14

Figure 1: SF at the chemical potential $A(\mathbf{k}, \omega=0)$ in the first Brillouin zone for the $t-t^{\prime}-J$ model with $t^{\prime} / t=-0.3$. Results are shown for $T / t=0.1$ and three doping concentrations. Dots show points, where SF and $\Sigma^{\prime \prime}(\mathbf{k}, \omega)$ in Fig. 4 are presented.

in the quarter of the FBZ. As usual, such a plot is used to locate the FS at particular doping. It is well resolved, that at lowest doping $c_{h}=0.05$ (Fig. 1a) we obtain appreciable SF weight only in the nodal direction close to $\mathbf{k}_{K}=(\pi / 2, \pi / 2)$ which is clearly in agreement with experimental results for hole-doped cuprates at low doping [1, 2, 3]. Moving to the edge of the FBZ one enters the pseudogap region, i.e. a region where the QP peaks at the FS are strongly suppressed and the FS is in fact not well resolvable, at least not within our numerical limitations. While this behavior is most pronounced at the lowest $c_{h}$ in Fig. 1a, it persists also for higher but still 'underdoped' $c_{h}=0.1$ in Fig.1b. Evidently, the FS gradually builds up with doping, while the pseudogap remains most pronounced at the edge of the FBZ (antinodal region). On the other hand, at 'optimum' doping $c_{h} \sim 0.17$ in Fig. 1c the FS becomes almost a continuous line. Moreover, at the edge of the FBZ near $\mathbf{k}_{X}=(\pi, 0)$ the SF becomes even more pronounced than in the nodal direction, the effect indeed observed in LSCO and Na-CCOC at intermediate doping [2, 5]. A decrease in intensity between nodal and antinodal region is, however, more reminiscent of electrondoped $\mathrm{Nd}_{2-x} \mathrm{Ce}_{x} \mathrm{CuO}_{4}$ analogue [19]. It should be noted that the observed effective FS appears always larger than expected from the Luttinger theorem, where $V_{F S} / V_{B Z}=\left(1-c_{h}\right) / 2$. A deviation is recently established also from the systematic ARPES study of Na-CCOC [3], whereby our results show even larger discrepancy.
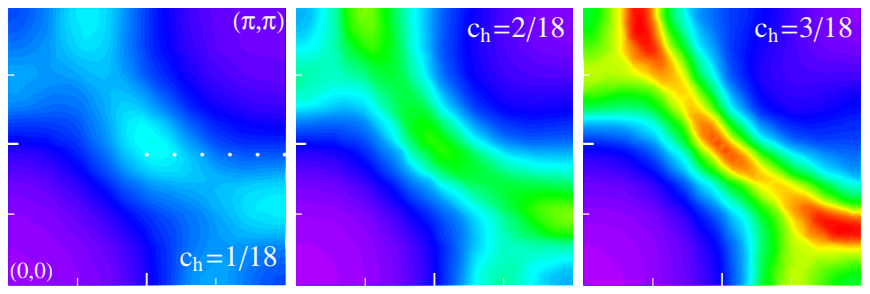

0 0.28

Figure 2: Same as Fig. 1 for the $t-J$ model.

For comparison we present in Fig. 2 also corresponding plots for the reference $t-J$ model. We detect here more con- 
tinuous FS line over the entire FBZ already in the low-doping regime. Still, the SF weight is much weaker in the latter regime, similar to Fig. 1a. The effect of increasing doping is clearly visible in the sharper and better defined FS due to the increasing weight of the SF at the FS. The deviation from the Luttinger theorem is still observable, although it is smaller relative to Fig. 1. The difference between the SF in Figs. 1,2 can be at lowest doping $c_{h} \sim 0.05$ in a naive way explained via the rigid band picture and an effective dispersion $\epsilon(\mathbf{k})$ of a single hole in an AFM [20]. While within the $t-J$ model such a dispersion is very anisotropic around the $\mathbf{k}_{K}$, leading to a minor difference $\Delta \epsilon=\epsilon\left(\mathbf{k}_{K}\right)-\epsilon\left(\mathbf{k}_{X}\right), t^{\prime}<0$ induces more isotropic dispersion around $\mathbf{k}_{K}$ and therefore more truncated FS. On the other hand, at intermediate doping FS already appears as a large one with the dispersion according to the renormalized 'free' band where $t^{\prime}$ directly influences its curvature.

One of the clearest manifestations of the pseudogap is the DOS $\mathcal{N}(\omega)=(2 / N) \sum_{\mathbf{k}} A(\mathbf{k}, \omega)$, as presented in Fig. 3, both for $t^{\prime} / t=-0.3$ and $t^{\prime}=0$. We note that the results for $t-J$ model are quite close to previous ones [16], obtained without the $\mathrm{BC}$ averaging. This confirms that the pseudogap, more or less pronounced at $\omega \sim 0$, is a robust feature of the model. Already from DOS one can conclude, that pseudogap vanishes with increased doping while $t^{\prime}<0$ enhances it. E.g., for $t^{\prime}<0$ DOS at $\omega \sim 0$ is reduced due to less coherent band and the pseudogap remains more pronounced up to $c_{h} \sim 0.17$.

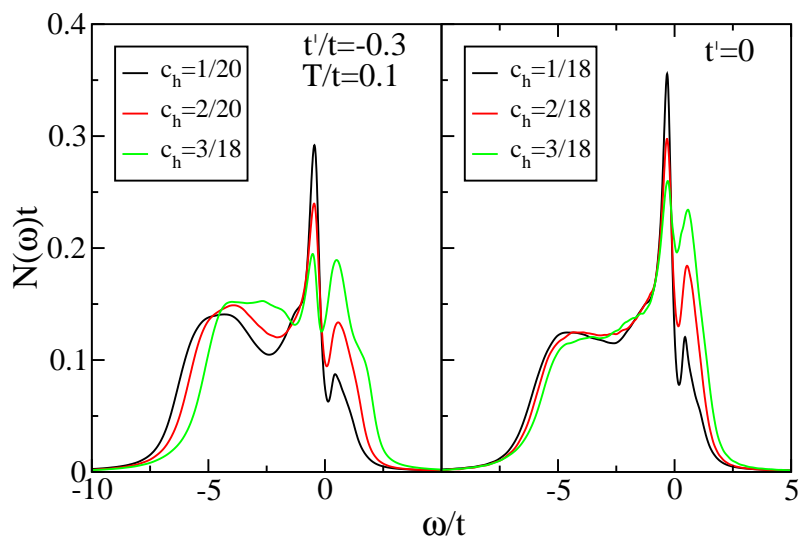

Figure 3: Density of states $\mathcal{N}(\omega)$ at different dopings $c_{h}$ for: a) $t^{\prime} / t=-0.3$ and $\left.\mathrm{b}\right) t^{\prime}=0$.

However, DOS is not selective enough to reveal the origin and k-dependence of the pseudogap. Hence, we present in Fig. 4 sets of SF and corresponding self-energies $\Sigma^{\prime \prime}(\mathbf{k}, \omega)$ taken along the paths shown in Fig. 1 for each doping, respectively. We choose points, being slightly below the FS in the nodal part (as this does not lead to an essential change in $\left.\Sigma^{\prime \prime}(\mathbf{k}, \omega)\right)$ and matching the pseudogapped FS at the edge of the FBZ.

Fig. 4a shows SF $A(\mathbf{k}, \omega)$ and $\Sigma^{\prime \prime}(\mathbf{k}, \omega)$ for $t^{\prime} / t=-0.3$ at lowest doping $c_{h}=0.05$. We notice that along the chosen path a sharper peak in the nodal region, corresponding approximately to a brighter spot of large weight in Fig. 1a, develops into a pronounced two peak structure in the antinodal region as a manifestation of the pseudogap in SF. Besides that, SF shows well known incoherent and nondispersive part for $\omega \ll 0$ and in this respect strong asymmetry between $\omega<0$ and $\omega>0$ regions, well evident also in $\mathcal{N}(\omega)$ in Fig. 3. The latter features persist up to high doping. In addition, from Fig. 4 it follows that the pseudogap splitting near $\mathbf{k}_{X}$ closes on doping, whereby the QP become better defined although still heavily damped.
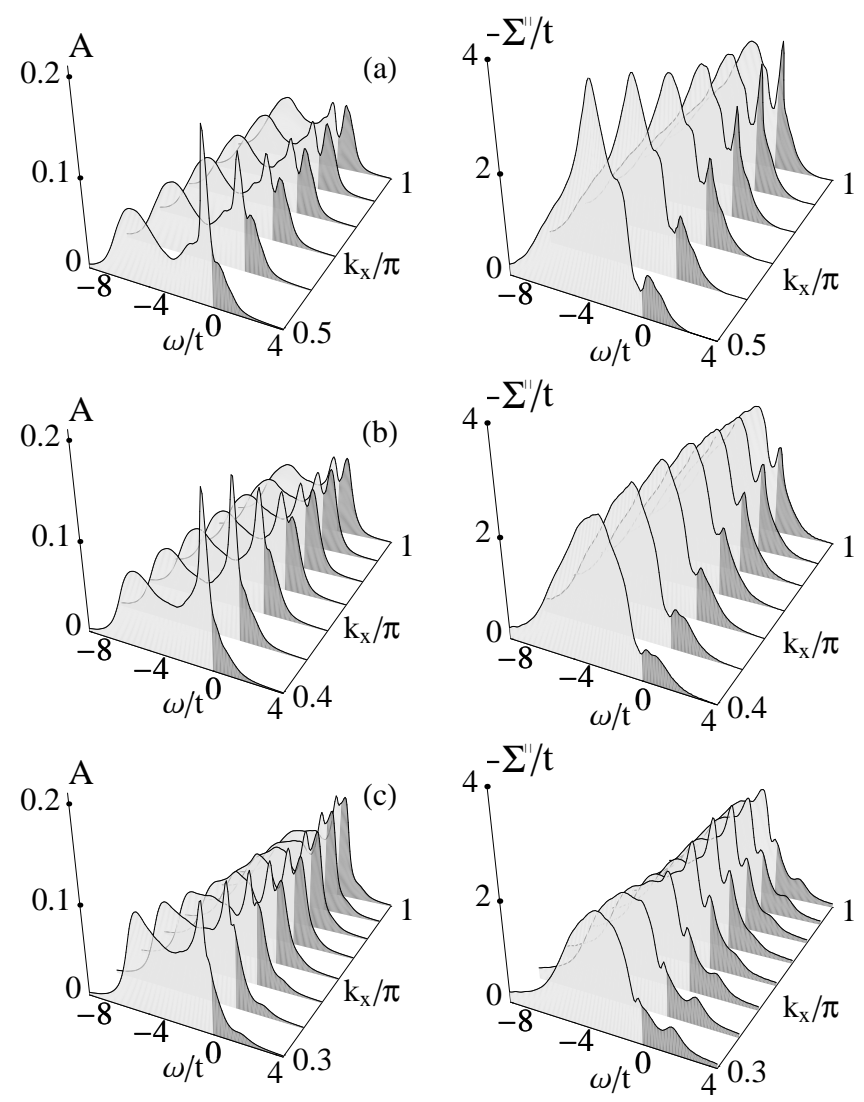

Figure 4: Spectral function $A(\mathbf{k}, \omega)$ (left column) and corresponding $\Sigma^{\prime \prime}(\mathbf{k}, \omega)$ (right column) for $t^{\prime} / t=-0.3$ taken in $\mathbf{k}$-points shown in Fig. 1 for dopings $c_{h}=0.05,0.1,0.17$, respectively.

A sensitive probe of QP damping and pseudogap behavior are self energies $\Sigma^{\prime \prime}(\mathbf{k}, \omega)$ in Fig. 4 revealing several features: a) at $\omega \ll 0$ large and broad damping function $\left|\Sigma^{\prime \prime}\right| \gg t$ is consistent with the incoherent part of SF, b) much smaller damping for $\omega \gg 0$, c) linear behavior $\Sigma^{\prime \prime}(\mathbf{k}, \omega \lesssim 0)$ as described within the MFL scenario, d) a pronounced peak of a Lorentzian form is building-up at $\omega \sim 0$ as we move to the antinodal part of FS, representing a clear signature of the pseudogap. Namely, a sharp peak $\left|\Sigma^{\prime \prime}\right| \propto \pi \Delta^{2} \delta(\omega)$ would open a real gap in the SF at the FS, while broader one opens the pseudogap, with the effective peak splitting $E_{P G} \sim 2 \Delta$.

It follows from Fig. 4 that the pseudogap contribution in $\Sigma^{\prime \prime}(\mathbf{k}, \omega)$ for $t^{\prime} / t=-0.3$ is largest in the antinodal region and 


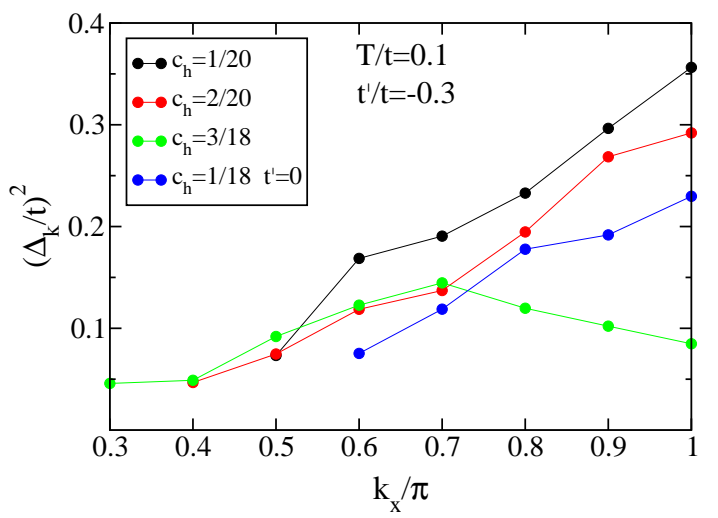

Figure 5: Momentum dependence of the pseudogap weight $\Delta_{\mathrm{k}}^{2}$ obtained for $t^{\prime} / t=-0.3$ and $t^{\prime}=0$ for different $c_{h}$ along the paths presented in Figs. 1,2.

for lowest doping $c_{h}=0.05,0.1$, where it is also very sharp. Elsewhere it loses weight and becomes indistinguishable from the background. In order to quantify $\Sigma^{\prime \prime}(\mathbf{k}, \omega)$ close to FS and to study its momentum dependence we assume for $\omega \lesssim 0$ the form

$$
\Sigma(\mathbf{k}, \omega) \sim \Sigma_{M F L}(\mathbf{k}, \omega)+\Delta_{\mathbf{k}}^{2} /\left(\omega-\omega_{\mathbf{k}}^{*}+i \Gamma_{\mathbf{k}}\right) .
$$

whereby the MFL part is taken as $-\Sigma_{M F L}^{\prime \prime}(\mathbf{k}, \omega \sim 0) \sim a_{\mathbf{k}}+$ $b_{\mathbf{k}}|\omega|$. We use Eq. (4) to fit the calculated $\Sigma^{\prime \prime}(\mathbf{k}, \omega)$ within the range $-t<\omega<0.2 t$. The latter is chosen in this way since at $\omega<0$ the linear part extends at least down to $\omega \sim-t$ (at $c_{h}=0.17$ even to $\omega \sim-2 t$ ) while the position of the peak $\omega_{\mathbf{k}}^{*}<0.2 t$ and the linear part at $\omega>0$ is also very restricted. Moreover, the fit and the pseudogap interpretation is meaningful only as far as $\Gamma_{\mathbf{k}}<\Delta_{\mathbf{k}}$.

The fitting analysis using Eq. (4) yields results presented in Fig. 4. Again, the variation along the paths shown in Figs. 1,2 is considered, whereby only points with $\Gamma_{\mathbf{k}}<\Delta_{\mathbf{k}}$ are presented. We observe for $t^{\prime} / t=-0.3$, as expected from Fig. 3, a clear decrease of $\Delta_{\mathrm{k}}$ towards the nodal direction for $c_{h}=0.05,0.1$, whereas for 'optimum' $c_{h}=0.17$ the weight is reduced in particular in the antinodal region with a maximum at an intermediate $\mathbf{k}$ along the path. The $t$ - $J$ model reveals smaller $\Delta_{\mathrm{k}}$, in particular it is hardly resolved for larger $c_{h}=0.11,0.17$, hence we omit these data in Fig. 4 .

As expected, $b_{\mathbf{k}}$ shows much less pronounced momentum and even doping dependence. The linear regime is particularly broad for the 'optimum' doping $c_{h}=0.17$, where we find $b_{\mathbf{k}}=1.45 \pm 0.2,1.2 \pm 0.2$ for $t^{\prime} / t=-0.3$ and $t^{\prime}=0$, respectively. In order to make a quantitative comparison with experimentally determined $\tilde{b} \sim 0.8$ [9] one can compare only the effective damping $Z_{\mathbf{k}}\left|\Sigma^{\prime \prime}(\mathbf{k}, \omega)\right|$ whereby $Z_{\mathbf{k}}$ is the QP weight well defined only in the region without pseudogap. In ARPES, $Z_{\mathbf{k}}$ is less clearly defined and is taken $Z_{\mathbf{k}} \sim 0.5$ [9], while we extract $Z_{\mathbf{k}} \sim 0.3 \pm 0.1$. This brings model $b_{\mathbf{k}}$ and experimental $\tilde{b}$ close together.

Similar results we get for lower $c_{h} \leq 0.1$, i.e. $b_{\mathbf{k}}=$ $1.8 \pm 0.2,1.5 \pm 0.2$ for $t^{\prime} / t=-0.3$ and $t^{\prime}=0$, respectively.
However, the range of linearity shrinks for low doping compatible with less justified MFL form for $\omega \sim 0$. Furtheron, the MFL scenario predicts $a \sim \pi b T$ [8]. As our results are calculated at $T=0.1 t$, we expect the MFL contribution $a \sim 0.5 t$. For the intermediate doping $c_{h}=0.17$ and $t^{\prime}=-0.3 t$ we indeed find $a_{\mathbf{k}}$ close to this value along the nodal direction, with the increasing tendency towards the edge of the FBZ where $a_{\mathbf{k}} \sim t$. This is consistent with the analysis of ARPES in Bi2212 where in the antinodal region $\tilde{a}_{\mathbf{k}} \sim 200 \mathrm{meV}$ has been observed [9]. However, within our approach a more detailed analysis of $a_{\mathbf{k}}$ is difficult since it is hard to separate it from the pseudogap contribution, in particular at lower doping, where the pseudogap part dominates. Furthermore, our results indicate that $a_{\mathbf{k}}$ and the pseudogap contribution could become the same feature when $\Gamma>\Delta$. Similar question can arise also in the interpretation of $\mathrm{Bi} 2212$ data where the pseudogap appears to be present as well [9].

In conclusion, we have shown that within the $t-t^{\prime}-J$ model as relevant for hole-doped cuprates the self energy $\Sigma(\mathbf{k}, \omega)$ can be decomposed into two parts. $\Sigma_{M F L}(\mathbf{k}, \omega)$ is almost $\mathbf{k}$-independent as well as weakly changing with doping and $t^{\prime}$, but still anomalous and following the MFL scenario. Another contribution generating the pseudogap is strongly $\mathbf{k}$ dependent most pronounced in the antinodal part of the FBZ and at low doping. It is much more expressed for $t^{\prime}<0$ case, which is more relevant for hole-doped cuprates. It seems that at intermediate doping the pseudogap contribution transforms into a constant part of QP damping $a_{\mathbf{k}}$ which therefore becomes k-dependent.

It should be noted that the pseudogap scale discussed here is the large (high-energy) one [1] as e.g. very evident in LSCO [2, 5] and Na-CCOC [3] at low doping. The latter is responsible for effective truncation of the FS in this regime, as observed in our results in Figs. 1,2. We also emphasize that our analysis as well as experimental does not give a clear answer whether such a pseudogap indeed vanishes in the nodal direction, or the weak coherent QP peak appears inside the pseudogap. As far as the origin of these phenomena is concerned, $\mathbf{k}$ and $t^{\prime}$ dependence of the pseudogap as well as underlying $\Sigma_{M F L}(\omega)$ are in favor of the interpretation in terms of coupling to short-range AFM spin fluctuations [11, 13, 18].

We acknowledge useful discussions with T. Tohyama. This work was supported by the Slovenian Research Agency under grant Pl-0044.

[1] for a review see A. Damascelli, Z. Hussain, and Z.-X. Shen, Rev. Mod. Phys. 75, 473 (2003).

[2] T. Yoshida et al., cond-mat/0510608

[3] K. M. Shen et al., Science 307, 910 (2005).

[4] D. S. Marshall et al., Phys. Rev. Lett. 76, 4841 (1996).

[5] A. Ino et al., Phys. Rev. Lett. 81, 2124 (1998); A. Ino et al., Phys. Rev. B 62, 4137 (2000).

[6] T. Yoshida et al., Phys. Rev. Lett. 91, 027001 (2003).

[7] T. Valla et al., Science 285, 2110 (1999). 
[8] C. M. Varma, P. B. Littlewood, S. Schmitt-Rink, E. Abrahams, and A. E. Ruckenstein, Phys. Rev. Lett. 63, 1996 (1989).

[9] A. Kaminski et al., Phys. Rev. B 71, 014517 (2005).

[10] R. Preuss, W. Hanke, C. Gröber, and H. G. Evertz, Phys. Rev. Lett. 79, 1122 (1997).

[11] A. Macridin, M. Jarrell, Th. Maier, and P. R. C. Kent, cond-mat/0509166

[12] C. Huscroft, M. Jarrell, Th. Maier, S. Moukouri and A. N. Tahvildarzadeh, Phys. Rev. Lett. 86, 139 (2001).

[13] B. Kyung, S. S. Kancharla, D. Senechal, A. - M. S. Tremblay, M. Civelli, and G. Kotliar, Phys. Rev. B 73, 165114 (2006).

[14] M. Civelli, M. Capone, S. S. Kancharla, O. Parcollet, and G.
Kotliar, Phys. Rev. Lett. 95, 106402 (2005).

[15] for a review see J. Jaklič and P. Prelovšek, Adv. Phys. 49, 1 (2000).

[16] J. Jaklič and P. Prelovšek, Phys. Rev. B 55, R7307 (1997); P. Prelovšek, J. Jaklič, and K. Bedell, Phys. Rev. B 60, 40 (1999).

[17] T. Tohyama, Phys. Rev. B 70, 174517 (2004).

[18] P. Prelovšek and A. Ramšak, Phys. Rev. B 63, 180506(R) (2001).

[19] N. P. Armitage et al., Phys. Rev. Lett. 88, 257001 (2002).

[20] A. Nazarenko, K. J. E. Vos, S. Haas, E. Dagotto, and R. J. Gooding, Phys. Rev. B 51, 8676 (1995). 